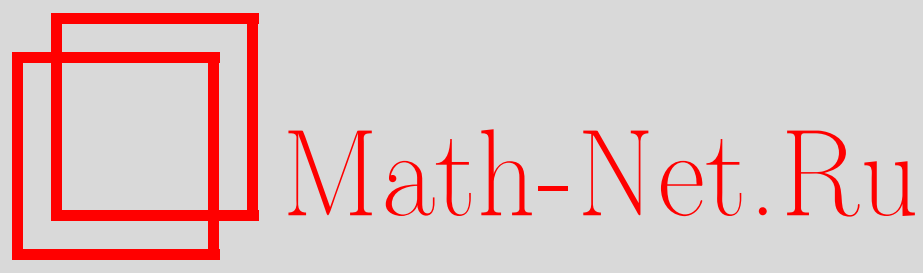

Р. Н. Гарифуллин, А. В. Михайлов, Р. И. Ямилов, Дискретное уравнение на квадратной решетке с нестандартной структурой высших симметрий, ТМФ, 2014, том 180, номер 1, 17-34

DOI: https://doi.org/10.4213/tmf8663

Использование Общероссийского математического портала Math-Net.Ru подразумевает, что вы прочитали и согласны с пользовательским соглашением http: //www . mathnet.ru/rus/agreement

Параметры загрузки:

IP : 54.198.55.26

26 апреля 2023 г., 15:47:27

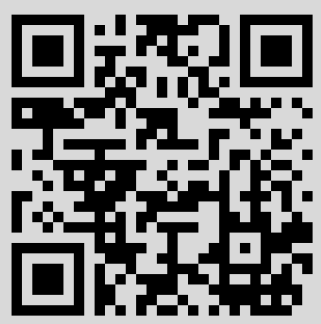




\title{
ФИЗИКА
}

Том 180, № 1

июль, 2014

(C) 2014 г. Р. Н. Гарифуллин*, А. В. Михайлов ${ }^{\dagger}$, Р. И. Ямилов*

\section{ДИСКРЕТНОЕ УРАВНЕНИЕ НА КВАДРАТНОЙ РЕШЕТКЕ С НЕСТАНДАРТНОЙ СТРУКТУРОЙ ВЫСШИХ СИММЕТРИЙ}

\begin{abstract}
Уточнена природа интегрируемости недавно найденного дискретного уравнения на квадратной решетке, обладающего нестандартной симметрийной структурой. Найдена его $L-A$-пара и показано, что она также является необычной. Для этого дискретного уравнения построены иерархии высших симметрий и законов сохранения, из этого уравнения получены две интегрируемые системы гиперболического типа. Иерархии высших симметрий и законов сохранения также оказываются нестандартными по сравнению с известными уравнениями этого класса.
\end{abstract}

Ключевые слова: дискретные интегрируемые уравнения, высшие симметрии, законы сохранения, $L-A$-пара.

DOI: $10.4213 / \operatorname{tmf} 8663$

\section{1. ВВЕДЕНИЕ}

В работе [1] было найдено уравнение

$$
u_{n+1, m+1}\left(u_{n, m}-u_{n, m+1}\right)-u_{n+1, m}\left(u_{n, m}+u_{n, m+1}\right)+2=0,
$$

где $n, m$ - произвольные целые числа. Было показано, что его высшая симметрия в направлении $m$ имеет вид

$$
\frac{d}{d t_{2}} u_{n, m}=(-1)^{n} \frac{u_{n, m+1} u_{n, m-1}+u_{n, m}^{2}}{u_{n, m+1}+u_{n, m-1}} .
$$

Простейшая высшая симметрия в направлении $n$ оказывается уравнением следующего вида:

$$
\frac{d}{d t_{1}} u_{n, m}=h_{n, m} h_{n-1, m}\left(a_{n} u_{n+2, m}-a_{n-1} u_{n-2, m}\right),
$$

* Институт математики с ВЦ УНЦ РАН, Уфа, Россия.

E-mail: rustem@matem.anrb.ru, RvlYamilov@matem.anrb.ru

${ }^{\dagger}$ University of Leeds, Department of Applied Mathematics, Leeds, UK.

E-mail: A.V.Mikhailov@leeds.ac.uk 
где

$$
h_{n, m}=u_{n+1, m} u_{n, m}-1, \quad a_{n+2}=a_{n} .
$$

Эта симметрия зависит от произвольной двухпериодической функции $a_{n}$, которая может быть представлена следующим образом:

$$
a_{n}=\tilde{a}+\hat{a}(-1)^{n}
$$

где $\tilde{a}, \hat{a}$ - произвольные комплексные числа. Мы имеем здесь как автономный частный случай $a_{n}=1$, так и неавтономный случай $a_{n}=(-1)^{n}$, все остальные возможные частные случаи являются их линейными комбинациями. Например, мы можем получить:

$$
a_{n}=\frac{1+(-1)^{n}}{2}= \begin{cases}0, & n=2 k+1, \\ 1, & n=2 k\end{cases}
$$

Высшие симметрии (2), (3) сами по себе являются интегрируемыми уравнениями. Более точно, при каждом фиксированном $n$ в случае (2) и любом фиксированном $m$ в случае (3) мы имеем интегрируемое уравнение с одной непрерывной и одной дискретной переменной. Уравнение (1) порождает цепочки автопреобразований Беклунда для каждого из уравнений (2), (3) (подробнее см. в [2]). Симметрии (2), (3) совместны не только с уравнением (1), но и друг с другом на решениях дискретного уравнения (1). С другой стороны, уравнение (1) может быть получено как условие совместности высших симметрий (2), (3). По этим и многим другим причинам в работе рассматривается тройка уравнений (1)-(3) в совокупности вместо одного дискретного уравнения (1). Такой подход позволяет получить некие важные результаты, представленные в разделах 2,3 .

Практически все известные интегрируемые дискретные уравнения вида

$$
F_{n, m}\left(u_{n, m}, u_{n+1, m}, u_{n, m+1}, u_{n+1, m+1}\right)=0,
$$

у которых существуют симметрии в каждом из направлений $n$ и $m$, имеют высшие симметрии вида [2]-[5]

$$
\begin{aligned}
\frac{d}{d t_{1}} u_{n, m} & =\Phi_{n, m}\left(u_{n+1, m}, u_{n, m}, u_{n-1, m}\right), \\
\frac{d}{d t_{2}} u_{n, m} & =\Psi_{n, m}\left(u_{n, m+1}, u_{n, m}, u_{n, m-1}\right) .
\end{aligned}
$$

Помимо уравнения (1) нам известны только несколько исключений, полученных в работах [6]-[8]. В этих примерах простейшие высшие симметрии в обоих направлениях имеют более сложную структуру, чем (7): они зависят также от $u_{n \pm 2, m}$ или $u_{n, m \pm 2}$.

С точки зрения структуры высших симметрий уравнение (1) является единственным в своем роде, поэтому мы изучим его более детально. В разделе 2 мы строим $L-A$-пары для каждого из уравнений $(1)-(3)$ и демонстрируем, что $L-A$-пара уравнения (1) также является нестандартной. В разделе 3 , используя полученную тройку $L-A$-пар, мы находим для дискретного уравнения (1) две иерархии законов 
сохранения. В разделе 4 построены мастер-симметрия для уравнения (2) и оператор рекурсии для уравнения (3), таким образом мы получаем иерархии высших симметрий дискретного уравнения в каждом из направлений $n$ и $m$. В разделе 5 с использованием тройки уравнений (1)-(3) мы построили два примера непрерывных интегрируемых гиперболических систем.

\section{2. $L-A$-ПАРЫ}

При построении $L-A$-пары уравнения (1) мы используем следующее интересное свойство симметрии (3). Оказывается, она эквивалентна известной системе двух уравнений, найденной Цучидой (см. [9], (3.13)), и $L-A$-пара для этой системы известна и приводится в той же статье.

Система Цучиды после преобразования

$$
v_{k} \rightarrow(-1)^{k} v_{k}, \quad w_{k} \rightarrow(-1)^{k+1} w_{k}
$$

и добавления точечной симметрии может быть записана в виде

$$
\begin{aligned}
\frac{d}{d t_{1}} v_{k} & =\left(\alpha v_{k+1}-\beta v_{k-1}\right)\left(v_{k} w_{k}-1\right)\left(v_{k} w_{k+1}-1\right), \\
\frac{d}{d t_{1}} w_{k} & =\left(\beta w_{k+1}-\alpha w_{k-1}\right)\left(v_{k} w_{k}-1\right)\left(v_{k-1} w_{k}-1\right) .
\end{aligned}
$$

Эта система является интегрируемой дискретизацией одного из нелинейных уравнений Шредингера с производной, введенного в работе [10] (см. также [11]). Уравнение (3) при любом фиксированном $m$ связано с системой (8) следующим образом:

$$
v_{k}=u_{2 k, m}, \quad w_{k}=u_{2 k-1, m}, \quad \alpha=a_{2 k}, \quad \beta=a_{2 k-1} .
$$

Отметим, что система (8) является линейной комбинацией двух совместных систем, соответствующих частным случаям $\alpha=1, \beta=0$ и $\alpha=0, \beta=1$. В этом смысле система (8) аналогична известной цепочке Абловица-Ладика, которая также является линейной комбинацией двух коммутирующих уравнений типа релятивистской цепочки Тоды (см., например, п. 5.2 в работе [12]).

Уравнения, связанные преобразованием (9), эквивалентны, и мы можем переносить обобщенные симметрии и законы сохранения с одного уравнения на другое (см. [13]). Мы получаем $L-A$-пару для уравнения (3) с помощью преобразования (9), переписывая известную $L-A$-пару уравнения (8), найденную в работе [9]. Эта $L-A$-пара стандартна и задается следующей парой совместных линейных уравнений:

$$
T_{k} \Phi_{k}=U_{k} \Phi_{k}, \quad D_{t_{1}} \Phi_{k}=V_{k} \Phi_{k}
$$

где $\Phi_{k}$ является двухкомпонентной вектор-функцией, $U_{k}, V_{k}-(2 \times 2)$-матрицы, зависящие от спектрального параметра, $T_{k}$ - оператор сдвига по переменой $k$ : $T_{k} h_{k}=h_{k+1}$.

Из преобразования (9) видно, что сдвиг $v_{k}, w_{k}$ по переменной $k$ соответствует двойному сдвигу $u_{n, m}$ по $n$. Поэтому мы получаем $L-A$-пару для уравнения $(3)$ в несколько иной форме:

$$
T_{n}^{2} \Psi_{n, m}=N_{n, m} \Psi_{n, m}, \quad D_{t_{1}} \Psi_{n, m}=A_{n, m} \Psi_{n, m} .
$$


Здесь $T_{n}$ - оператор сдвига по переменной $n$, а матрицы $N_{n, m}, A_{n, m}$ имеют вид

$$
\begin{aligned}
& N_{n, m}=\left(\begin{array}{cc}
h_{n, m}(1-\lambda)-2 \lambda & u_{n+1, m}(\lambda-1) \\
-2 \lambda u_{n, m} h_{n+1, m} & h_{n+1, m}(\lambda-1)
\end{array}\right), \\
& A_{n, m}=\left(\begin{array}{rr}
h_{n-1, m}\left(a_{n-1} u_{n+1, m} u_{n-2, m}+a_{n} \frac{\lambda-1}{\lambda+1}\right)+ & \\
+a_{n-1} \frac{2 \lambda}{\lambda-1} & -a_{n} u_{n-1, m} \frac{\lambda-1}{\lambda+1}-a_{n-1} u_{n+1, m} \\
2 \lambda h_{n-1, m}\left(\frac{u_{n, m} a_{n}}{1+\lambda}+\frac{u_{n-2, m} a_{n-1}}{\lambda-1}\right)- & \\
-a_{n} u_{n, m} u_{n-1, m} \frac{2 \lambda}{1+\lambda} & h_{n, m}\left(a_{n} u_{n-1, m} u_{n+2, m}-a_{n-1}\right)
\end{array}\right) .
\end{aligned}
$$

Оператор $T_{n}^{2}-N_{n, m}$, возникающий в первом из уравнений (11), является дискретным аналогом оператора Шредингера с матричными коэффициентами. Условие совместности уравнений (11) имеет вид

$$
D_{t_{1}} N_{n, m}=\left(T_{n}^{2} A_{n, m}\right) N_{n, m}-N_{n, m} A_{n, m},
$$

и это соотношение эквивалентно уравнению (3). В терминах $(4 \times 4)$-матриц $L-A$-пара может быть переписана в стандартном виде:

$$
\begin{aligned}
T_{n}\left(\begin{array}{c}
\Psi_{n, m} \\
\Psi_{n+1, m}
\end{array}\right) & =\left(\begin{array}{cc}
0 & E \\
N_{n, m} & 0
\end{array}\right)\left(\begin{array}{c}
\Psi_{n, m} \\
\Psi_{n+1, m}
\end{array}\right), \\
D_{t_{1}}\left(\begin{array}{c}
\Psi_{n, m} \\
\Psi_{n+1, m}
\end{array}\right) & =\left(\begin{array}{cc}
A_{n, m} & 0 \\
0 & A_{n+1, m}
\end{array}\right)\left(\begin{array}{c}
\Psi_{n, m} \\
\Psi_{n+1, m}
\end{array}\right) .
\end{aligned}
$$

Что касается симметрии (2), то при любом фиксированном $n \in \mathbb{Z}$, переходя к $z_{m}=i^{m} u_{n, m}$ и используя растяжение времени, мы получаем хорошо известное уравнение [14], [15]

$$
\frac{d z_{m}}{d t_{2}}=\frac{z_{m+1} z_{m-1}+z_{m}^{2}}{z_{m+1}-z_{m-1}} .
$$

Для уравнений этого типа вспомогательная линейная задача известна [4] и является стандартной:

$$
T_{m} \Psi_{n, m}=M_{n, m} \Psi_{n, m}, \quad D_{t_{2}} \Psi_{n, m}=B_{n, m} \Psi_{n, m} .
$$

Здесь $M_{n, m}\left(u_{n, m}, u_{n, m+1}\right), B_{n, m}\left(u_{n, m-1}, u_{n, m}, u_{n, m+1}\right)-(2 \times 2)$-матрицы, и условие совместности имеет вид

$$
D_{t_{2}} M_{n, m}=\left(T_{m} B_{n, m}\right) M_{n, m}-M_{n, m} B_{n, m} .
$$

Первые линейные дискретные уравнения из (11), (16) задают пару Лакса для дискретного уравнения (1), если в них совпадают вектор-функции $\Psi_{n, m}$ и спектральные параметры $\lambda$. После замены вектор-функции с помощью матрицы $\Omega_{n, m}$ матрица $M_{n, m}$ изменяется в соответствии с калибровочным преобразованием

$$
\widetilde{M}_{n, m}=\Omega_{n, m+1}^{-1} M_{n, m} \Omega_{n, m} .
$$

Матрица $\Omega_{n, m}$ и замена спектрального параметра могут быть найдены прямым вычислением, но мы предпочитаем другой, более простой путь. Мы используем соотношение

$$
\left(T_{n}^{2} M_{n, m}\right) N_{n, m}=\left(T_{m} N_{n, m}\right) M_{n, m}
$$


эквивалентное $(1)$, известную матрицу $N_{n, m}$ и ищем матрицу $M_{n, m}\left(u_{n, m}, u_{n, m+1}\right)$. В результате мы получаем

$$
M_{n, m}=\left(\begin{array}{cc}
\lambda & \frac{1-\lambda}{u_{n, m}+u_{n, m+1}} \\
\lambda\left(u_{n, m+1}-u_{n, m}\right) & \frac{\lambda\left(u_{n, m}-u_{n, m+1}\right)}{u_{n, m}+u_{n, m+1}}
\end{array}\right) .
$$

Соответствующая матрица $B_{n, m}$, задающая $L$-A-пару для уравнения $(2)$, строится при помощи условия (17):

$$
\begin{aligned}
B_{n, m}= & \frac{(-1)^{n}}{u_{n, m+1}+u_{n, m-1}} \times \\
& \times\left(\begin{array}{cc}
(1-\lambda)\left(u_{n, m-1}-u_{n, m}\right) & 1-\lambda \\
\lambda\left(u_{n, m}^{2}-u_{n, m+1}^{2}\right) & \lambda\left(u_{n, m}+u_{n, m+1}\right)-\left(u_{n, m}+u_{n, m-1}\right)
\end{array}\right) .
\end{aligned}
$$

Отметим, что дискретная $L-A$-пара (18) тоже может быть переписана в стандартном виде:

$$
\left(T_{n} \widetilde{M}_{n, m}\right) \widetilde{N}_{n, m}=\left(T_{m} \widetilde{N}_{n, m}\right) \widetilde{M}_{n, m}
$$

в терминах $(4 \times 4)$-матриц $\widetilde{M}_{n, m}, \widetilde{N}_{n, m}$ блочной структуры. Сформулируем полученные результаты.

Теорема 1. L-A-пары для уравнений (1)-(3) задаются соответственно соотношениями (18), (17), (14), в которых $(2 \times 2)$-матрицы имеют вид (12), (13), (19), (20).

Практически для всех известных интегрируемых дискретных уравнений вида (6) $L$-A-пара задается соотношением $(21)$ с $(2 \times 2)$-матрицами $\widetilde{M}_{n, m}, \widetilde{N}_{n, m}$. Известны два исключения [6], [7], в которых $L-A$-пара имеет тот же вид (21), но задается матрицами размера $3 \times 3$. Эти уравнения являются дискретными аналогами уравнения Цицейки [16]

$$
u_{x y}=e^{u}+e^{-2 u},
$$

для которого $L-A$-пара определяется уравнениями

$$
D_{x} \Psi=L \Psi, \quad D_{y} \Psi=A \Psi
$$

с $(3 \times 3)$-матрицами $L, A[17]$. Наше уравнение $(1)$ является еще одним исключением: его $L$-A-пара $(18)$ задается $(2 \times 2)$-матрицами и может быть переписана в форме $(21)$ с матрицами размера $4 \times 4$.

Мы проверили, что дискретное уравнение (1) не имеет стандартной $L-A$-пары $(21)$ в терминах $(2 \times 2)$-матриц. Более точно, мы зафиксировали матрицу $\widetilde{M}_{n, m}=M_{n, m}$ из $(19)$ и искали $(2 \times 2)$-матрицу $\widetilde{N}_{n, m}\left(u_{n-1, m}, u_{n, m}, u_{n+1, m}, u_{n+2, m}\right)$. Используя эквивалентность $L-A$-пары (21) дискретному уравнению (1), мы показали, что матрицы $\widetilde{N}_{n, m}$ такого вида не существует.

Уравнение $(2)$ с $a_{n} \equiv 1$ при любом фиксированном $m \in \mathbb{Z}$ является автономной цепочкой вида

$$
\frac{d}{d t} u_{n}=G\left(u_{n-2}, u_{n-1}, u_{n}, u_{n+1}, u_{n+2}\right) .
$$


В этом классе уравнений оно занимает особое место, аналогичное месту уравнения (1) в классе (6). Большинство известных интегрируемых уравнений (22) являются высшими симметриями уравнений типа Вольтерра [14], [15] и имеют $L-A$-пары вида

$$
D_{t} L=\left(T_{n} A\right) L-L A
$$

с $(2 \times 2)$-матрицами $L, A$. Остальные известные нам интегрируемые цепочки являются аналогами уравнения Ито-Нариты-Богоявленского (см., например, [18]-[20])

$$
\frac{d}{d t} u_{n}=u_{n}\left(u_{n+2}+u_{n+1}-u_{n-1}-u_{n-2}\right)
$$

у которых $L-A$-пара (23) задается матрицами размера $3 \times 3$. Наше уравнение $(2)$ с $a_{n} \equiv 1$ имеет $L-A$-пару вида

$$
D_{t} L=\left(T_{n}^{2} A\right) L-L A
$$

с $(2 \times 2)$-матрицами. Она может быть переписана в виде $(23)$ с матрицами размеpa $4 \times 4$.

\section{3. ЗАКОНЫ СОХРАНЕНИЯ}

В этом разделе мы строим законы сохранения для дискретного уравнения (1), используя схему, предложенную Михайловым [21]. Этот подход предполагает существенное использование тройки совместных $L$ - $A$-пар $(18),(17),(14)$ для дискретного уравнения (1) и его высших симметрий (2), (3). Имеется альтернативный подход, который также позволяет решить эту задачу [22].

Метод, который мы используем для построения законов сохранения, предполагает формальную диагонализацию всех матриц, определяющих тройку $L-A$-пар (18), (17), (14). Причем удобно начинать эту диагонализацию с линейных дифференциальных уравнений, входящих в (11), (16). Для этого используются известные результаты теории линейных дифференциальных уравнений [23] (см. также [24]).

Вектор-функция $\Psi_{n, m}$ может быть преобразована с помощью матрицы $\Omega_{n, m}$ :

$$
\widetilde{\Psi}_{n, m}=\Omega_{n, m} \Psi_{n, m} .
$$

При этом матрицы, определяющие $L-A$-пары $(14),(17),(18)$, преобразуются по следующим формулам:

$$
\begin{aligned}
\widetilde{B}_{n, m} & =\Omega_{n, m}^{-1} B_{n, m} \Omega_{n, m}-\Omega_{n, m}^{-1} \partial_{t_{2}} \Omega_{n, m}, \\
\widetilde{M}_{n, m} & =\Omega_{n, m+1}^{-1} M_{n, m} \Omega_{n, m}, \\
\widetilde{N}_{n, m} & =\Omega_{n+2, m}^{-1} N_{n, m} \Omega_{n, m}, \\
\widetilde{A}_{n, m} & =\Omega_{n, m}^{-1} A_{n, m} \Omega_{n, m}-\Omega_{n, m}^{-1} \partial_{t_{1}} \Omega_{n, m} .
\end{aligned}
$$

Формальная диагонализация строится эффективно в окрестности полюсов матриц линейных дифференциальных операторов. Матрицы $B_{n, m}$ и $A_{n, m}$ имеют полюсы в точках $\lambda=\infty$ и $\lambda= \pm 1$ соответственно. Сформулируем лемму о диагонализации матрицы $B_{n, m}$ в окрестности ее полюса (см., например, [24], с. 86). 
ЛЕмма 1. Если главная часть $\partial B_{n, m} / \partial \lambda$ матрицъ $B_{n, m}$ имеет различные собственные значения, то существует формальный ряд

$$
\Omega_{n, m}=\Omega_{n, m}^{*}\left(E+\sum_{j=1}^{\infty} \lambda^{-j} \Omega_{n, m}^{(-j)}\right)
$$

такой, что $E$ - единичная матрица и $\Omega_{n, m}^{(-j)}, j \geqslant 1$, являются антидиагональными матрицами. При этом матрица $\widetilde{B}_{n, m}$, полученная при помощи первой из формул (26), имеет вид

$$
\widetilde{B}_{n, m}=\lambda B_{n, m}^{(1)}+\sum_{j=0}^{\infty} \lambda^{-j} B_{n, m}^{(-j)},
$$

где $B_{n, m}^{(l)}$ - диагональные матрицы.

Здесь мы имеем дело с формальными рядами. Вопросы сходимости этих рядов не обсуждаются. Для построения любого конечного числа законов сохранения достаточно знать конечное число коэффициентов формальных рядов.

Матрица $\partial B_{n, m} / \partial \lambda$ имеет различные собственные значения, поэтому существует преобразование, приводящее ее к диагональному виду. В качестве матрицы $\Omega_{n, m}^{*}$ можно взять любую из матриц этого преобразования, в частности

$$
\Omega_{n, m}^{*}=\left(\begin{array}{cc}
1 & 1 \\
u_{n, m}-u_{n, m-1} & u_{n, m}+u_{n, m-1}
\end{array}\right) .
$$

При этом находится $B_{n, m}^{(1)}$ :

$$
B_{n, m}^{(1)}=\left(\begin{array}{cc}
0 & 0 \\
0 & (-1)^{n+1}
\end{array}\right)
$$

Для нахождения матриц $\Omega_{n, m}^{(l)}$ и $B_{n, m}^{(l)}$ с $l \leqslant 0$ перепишем первое из уравнений (26) в виде

$$
\Omega_{n, m} \widetilde{B}_{n, m}=B_{n, m} \Omega_{n, m}-\partial_{t_{2}} \Omega_{n, m}
$$

и приравняем нулю коэффициенты при одинаковых степенях $\lambda$. Соотношение, соответствующее $\lambda^{1}$, выполняется тождественно за счет выбора $\Omega_{n, m}^{*}$ и $B_{n, m}^{(1)}$. Соотношения при других степенях $\lambda$ задают рекуррентные формулы для остальных коэффициентов $\widetilde{B}_{n, m}$ и $\Omega_{n, m}$, из которых эти коэффициенты находятся явным образом. В частности,

$$
\Omega_{n, m}^{(-1)}=\left(\begin{array}{cc}
0 & \frac{\left(u_{n, m+1}+u_{n, m+2}\right)\left(u_{n, m}-u_{n, m-1}\right)}{\left(u_{n, m}+u_{n, m-2}\right)\left(u_{n, m+1}+u_{n, m-1}\right)} \\
\frac{\left(u_{n, m}-u_{n, m-1}\right)\left(u_{n, m-1}-u_{n, m-2}\right)}{\left(u_{n, m}+u_{n, m-2}\right)\left(u_{n, m+1}+u_{n, m-1}\right)} & 0
\end{array}\right),
$$

другие коэффициенты мы не приводим по причине их громоздкости. 
Используя уравнение $(17)$ и формулу $(28)$ для $B_{n, m}^{(1)}$, мы можем доказать по индукции, что матрица $\widetilde{M}_{n, m}$ является диагональной. Из (26) мы находим

$$
\begin{aligned}
& \widetilde{M}_{n, m}=-\lambda \frac{u_{n, m+1}+u_{n, m-1}}{u_{n, m}+u_{n, m+1}}\left(\begin{array}{cc}
1 & 0 \\
0 & 0
\end{array}\right)- \\
& \quad-\left(\begin{array}{cc}
\frac{\left(u_{n, m+2}+u_{n, m+1}\right) u_{n, m-1}-u_{n, m}\left(u_{n, m+1}+u_{n, m+2}\right)}{\left(u_{n, m+2}+u_{n, m}\right)\left(u_{n, m}+u_{n, m+1}\right)} & 0 \\
0 & \frac{u_{n, m+1}-u_{n, m}}{u_{n, m}+u_{n, m+2}}
\end{array}\right)+\cdots .
\end{aligned}
$$

Используя уравнение (18) и коэффициент ряда $\widetilde{M}_{n, m}$ при $\lambda$, мы можем доказать при помощи индукции, что матрица $\widetilde{N}_{n, m}$ также является диагональной. Из (26) мы получаем

$$
\begin{aligned}
\tilde{N}_{n, m}= & -\lambda\left(\begin{array}{cc}
1+u_{n+1, m} u_{n, m-1} & 0 \\
0 & 1-u_{n+1, m} u_{n, m+1}
\end{array}\right)- \\
& -\frac{u_{n, m+1}-u_{n, m-1}}{u_{n, m+1}+u_{n, m-1}}\left(\begin{array}{cc}
1+u_{n+1, m} u_{n, m-1} & 0 \\
0 & -1+u_{n+1, m} u_{n, m+1}
\end{array}\right)+\cdots
\end{aligned}
$$

Матрицы $\widetilde{M}_{n, m}, \widetilde{N}_{n, m}$ диагональны, и их элементы являются формальными рядами по степеням $\lambda^{-1}$. Уравнение (18) для этих матриц может быть переписано в виде

$$
\left(T_{n}^{2}-1\right) \ln \widetilde{M}_{n, m}=\left(T_{m}-1\right) \ln \widetilde{N}_{n, m},
$$

причем мы используем обозначения

$$
\ln \left(\begin{array}{cc}
\alpha & 0 \\
0 & \beta
\end{array}\right)=\left(\begin{array}{cc}
\ln \alpha & 0 \\
0 & \ln \beta
\end{array}\right)
$$

Следующие диагональные элементы разлагаются в формальные ряды:

$$
\left(\ln \widetilde{M}_{n, m}\right)_{1,1}=\ln \lambda+\sum_{j=0}^{\infty} \lambda^{-j} p_{n, m}^{(j)}, \quad\left(\ln \widetilde{N}_{n, m}\right)_{1,1}=\ln \lambda+\sum_{j=0}^{\infty} \lambda^{-j} q_{n, m}^{(j)},
$$

и мы получаем иерархию законов сохранения:

$$
\left(T_{n}^{2}-1\right) p_{n, m}^{(j)}=\left(T_{m}-1\right) q_{n, m}^{(j)}, \quad j \geqslant 0 .
$$

Эти законы сохранения могут быть переписаны в стандартном виде:

$$
\left(T_{n}-1\right) p_{n, m}=\left(T_{m}-1\right) q_{n, m},
$$

поскольку $T_{n}^{2}-1=\left(T_{n}-1\right)\left(T_{n}+1\right)$.

Первые два закона сохранения выглядят следующим образом:

$$
\begin{array}{ll}
p_{n, m}^{(0)}=\ln \frac{u_{n, m+1}+u_{n, m-1}}{u_{n, m}+u_{n, m+1}}, & q_{n, m}^{(0)}=\ln \left(1+u_{n+1, m} u_{n, m+1}\right) ; \\
p_{n, m}^{(1)}=\frac{\left(u_{n, m+1}+u_{n, m+2}\right)\left(u_{n, m}-u_{n, m-1}\right)}{\left(u_{n, m+2}+u_{n, m}\right)\left(u_{n, m+1}+u_{n, m-1}\right)}, & q_{n, m}^{(1)}=\frac{u_{n, m+1}-u_{n, m-1}}{u_{n, m+1}+u_{n, m-1}} .
\end{array}
$$


Из вторых диагональных элементов мы получаем такие же законы сохранения (в соответствии с приведенным ниже комментарием), за исключением первого шага, на котором имеем

$$
\hat{p}_{n, m}^{(0)}=\ln \frac{u_{n, m}-u_{n, m+1}}{u_{n, m}+u_{n, m+2}}, \quad \hat{q}_{n, m}^{(0)}=\ln \left(1-u_{n+1, m} u_{n, m+1}\right) .
$$

Все плотности законов сохранения $p_{n, m}^{(j)}$ этой иерархии зависят от конечного числа функций из множества

$$
u_{n, m+k}, \quad k \in \mathbb{Z} .
$$

Для законов сохранения этого типа мы формулируем лемму 2 и определяем понятие порядка закона сохранения. Это позволяет нам различать законы сохранения между собой и выделять среди них тривиальные.

Определим сначала формальную вариационную производную в направлении $m$ от плотности $p_{n, m}$ :

$$
\frac{\delta_{m} p_{n, m}}{\delta_{m} u_{n, m}}=\sum_{k \in \mathbb{Z}} T_{m}^{-k} \frac{\partial p_{n, m}}{\partial u_{n, m+k}} .
$$

Эта сумма всегда конечна, так как $p_{n, m}$ зависит от конечного числа функций (32). Для любой сохраняющейся плотности $p_{n, m}$ имеем

$$
\frac{\delta_{m} p_{n, m}}{\delta_{m} u_{n, m}}=P_{n, m}\left(u_{n, m-M}, u_{n, m-M+1}, \ldots, u_{n, m+M-1}, u_{n, m+M}\right) .
$$

Используя функцию $r_{n, m}$, зависящую от конечного числа функций $(32)$, мы можем перейти к эквивалентному закону сохранения

$$
\left(T_{n}^{2}-1\right) \tilde{p}_{n, m}=\left(T_{m}-1\right) \tilde{q}_{n, m}
$$

по правилу

$$
\tilde{p}_{n, m}=p_{n, m}+\left(T_{m}-1\right) r_{n, m}, \quad \tilde{q}_{n, m}=q_{n, m}+\left(T_{n}^{2}-1\right) r_{n, m} .
$$

ЛЕмма 2. При помощи преобразования эквивалентности (33) плотность любого закона сохранения (30) может быть приведена к одному из следующих трех видов:

1) $\tilde{p}_{n, m}=0 \Leftrightarrow P_{n, m}=0$;

2) $\tilde{p}_{n, m}=\tilde{p}_{n, m}\left(u_{n, m}\right), \tilde{p}_{n, m}^{\prime} \not \equiv 0 \Leftrightarrow M=0, P_{n, m}\left(u_{n, m}\right) \not \equiv 0$;

3) $\tilde{p}_{n, m}=\tilde{p}_{n, m}\left(u_{n, m}, u_{n, m+1}, \ldots, u_{n, m+M}\right), \partial^{2} \tilde{p}_{n, m} / \partial u_{n, m} \partial u_{n, m+M} \not \equiv 0 \Leftrightarrow M>0$, $\partial P_{n, m} / \partial u_{n, m-M} \not \equiv 0, \partial P_{n, m} / \partial u_{n, m+M} \not \equiv 0$.

В случае 1 леммы 2 закон сохранения называется тривиальным. В случаях 2 и 3 мы называем его нетривиальным законом сохранения порядка $M$, мы будем писать ord $p_{n, m}=M$. Видно, что законы сохранения различного порядка не эквивалентны в смысле преобразования (33). Например, мы имеем следующее свойство: число переменных функции $p_{n, m}\left(u_{n, m}, u_{n, m+1}, \ldots, p_{n, m+k}\right), k>0$, может быть уменьшено с помощью преобразования (33) тогда и только тогда, когда $\partial^{2} p_{n, m} / \partial u_{n, m} \partial u_{n, m+k} \equiv 0$ для всех $n, m \in \mathbb{Z}$. 
Эта теория полностью аналогична теории для дискретно-дифференциальных законов сохранения вида

$$
D_{t} p_{m}=\left(T_{m}-1\right) q_{m} .
$$

Подробное обсуждение этой теории вместе с доказательствами приводится, например, в [13], [15]. Соответствующая теория для дискретных законов сохранения вида (31) обсуждается в работе [25].

Мы видим, что

$$
\operatorname{ord} p_{n, m}^{(0)}=2, \quad \operatorname{ord} p_{n, m}^{(1)}=3, \quad \text { ord } \hat{p}_{n, m}^{(0)}=2 .
$$

Таким образом, плотность $p_{n, m}^{(1)}$ отличается от двух других. Плотности $p_{n, m}^{(0)}$ и $\hat{p}_{n, m}^{(0)}$ могут быть, в принципе, эквивалентными с точностью до полной разности и умножения на константу. Однако если мы построим новый закон сохранения следующим образом:

$$
\begin{aligned}
& \check{p}_{n, m}^{(0)}=\hat{p}_{n, m}^{(0)}+p_{n, m}^{(0)}+\left(T_{m}-1\right)\left(p_{n, m}^{(0)}+\ln \left(u_{n, m}+u_{n, m+1}\right)\right)=\ln \frac{u_{n, m}-u_{n, m+1}}{u_{n, m}+u_{n, m+1}}, \\
& \check{q}_{n, m}^{(0)}=\hat{q}_{n, m}^{(0)}+q_{n, m}^{(0)}+\left(T_{n}^{2}-1\right)\left(p_{n, m}^{(0)}+\ln \left(u_{n, m}+u_{n, m+1}\right)\right)=\ln \left(h_{n, m} h_{n+1, m}\right),
\end{aligned}
$$

то мы увидим, что ord $\check{p}_{n, m}^{(0)}=1$. Таким образом, мы имеем три различных нетривиальных закона сохранения, порядок которых равен 1, 2 и 3.

Для построения законов сохранения в направлении $n$ используется линейное дифференциальное уравнение из (11) и диагонализация начинается с матрицы $A_{n, m}$. Матрица $A_{n, m}$ (13) имеет полюсы при $\lambda= \pm 1$, и мы можем провести формальную диагонализацию в терминах формальных рядов по степеням $\lambda+1$ или $\lambda-1$, воспользовавшись аналогом леммы 1 . В обоих случаях результаты совпадают, и мы ограничимся случаем $\lambda=-1$. В этом случае

$\Omega_{n, m}=\left(\begin{array}{cc}u_{n-1, m} & 1 \\ -h_{n-1, m} & u_{n, m}\end{array}\right)\left(\begin{array}{ccc}1 & & \frac{\lambda+1}{2} \frac{1-h_{n, m} h_{n+1, m}}{u_{n+1, m}}+\cdots \\ \frac{\lambda+1}{2} u_{n-3, m} h_{n-1, m} h_{n-2, m}+\cdots & 1\end{array}\right)$,

$\widetilde{N}_{n, m}=$

$=\left(\begin{array}{cc}2+(\lambda+1)\left(h_{n, m} u_{n-1, m} u_{n+2, m}-h_{n-1, m}-2\right) & 0 \\ 0 & -(\lambda+1) h_{n, m} h_{n+1, m}\end{array}\right)+\cdots$,

$\widetilde{M}_{n, m}=$

$=\left(\begin{array}{cc}1-(\lambda+1) \frac{u_{n, m}\left(1+u_{n, m+1} u_{n-1, m}\right)}{u_{n, m}+u_{n, m+1}} & 0 \\ 0 & \frac{u_{n, m+1}-u_{n, m}}{u_{n, m+1}+u_{n, m}}\left(1+(\lambda+1) \frac{h_{n-1, m} u_{n, m+1}}{u_{n, m+1}+u_{n, m}}\right)\end{array}\right)+\cdots$.

В главном члене разложения порядка $(\lambda+1)^{0}$ получаем закон сохранения (34), в следующих порядках $(\lambda+1)^{1}$ и $(\lambda+1)^{2}$ получаем законы сохранения

$$
\begin{aligned}
\check{p}_{n, m}^{(1)}= & \frac{2 h_{n-1, m} u_{n, m+1}}{u_{n, m+1}+u_{n, m}}, \\
\check{q}_{n, m}^{(1)}= & u_{n-1, m}\left(u_{n+2, m} h_{n, m}-u_{n, m}\right), \\
\check{p}_{n, m}^{(2)}= & 4 h_{n-1, m}\left(\frac{h_{n-1, m}\left(u_{n+2, m} h_{n, m}+u_{n, m}\right)}{u_{n, m}+u_{n, m+1}}-\frac{u_{n, m}^{2} h_{n-1, m}}{\left(u_{n, m}+u_{n, m+1}\right)^{2}}\right)+ \\
& +4 h_{n-1, m}-4 u_{n-1, m} u_{n+2, m} h_{n-1, m} h_{n, m},
\end{aligned}
$$




$$
\begin{aligned}
\check{q}_{n, m}^{(2)}= & 2 u_{n-1, m} u_{n+4, m} h_{n, m} h_{n+1, m} h_{n+2, m}+\left(h_{n-1, m} h_{n+1, m}-u_{n-1, m} u_{n+2, m}-1\right)^{2}- \\
& -2 h_{n-1, m} h_{n+1, m}-\left(h_{n+1, m}+2\right)^{2} .
\end{aligned}
$$

Таким способом мы можем получить иерархию законов сохранения вида (30), в которых функция $q_{n, m}^{(j)}$ зависит от конечного числа функций из множества

$$
u_{n+k, m}, \quad k \in \mathbb{Z} .
$$

Теория в этом случае аналогична той, которую мы обсуждали выше для законов сохранения в направлении $m$. Удобно переписать законы сохранения (30) в стандартной форме (31), где

$$
p_{n, m}=\left(T_{n}+1\right) p_{n, m}^{(j)}, \quad q_{n, m}=q_{n, m}^{(j)} .
$$

В этом случае функция $q_{n, m}$ играет роль плотности закона сохранения. Мы вводим формальную вариационную производную в направлении $n$ :

$$
\frac{\delta_{n} q_{n, m}}{\delta_{n} u_{n, m}}=\sum_{k \in \mathbb{Z}} T_{n}^{-k} \frac{\partial q_{n, m}}{\partial u_{n+k, m}}=Q\left(u_{n-N, m}, u_{n-N+1, m}, \ldots, u_{n+N, m}\right)
$$

и определяем порядок закона сохранения, как в предыдущем случае. Преобразование эквивалентности теперь имеет вид

$$
\tilde{q}_{n, m}=q_{n, m}+\left(T_{n}-1\right) s_{n, m}, \quad \tilde{p}_{n, m}=p_{n, m}+\left(T_{m}-1\right) s_{n, m},
$$

где $s_{n, m}$ зависит от конечного числа функций (35).

Для законов сохранения, приведенных выше, мы имеем

$$
\operatorname{ord} \check{q}_{n, m}^{(0)}=1, \quad \text { ord } \check{q}_{n, m}^{(1)}=3, \quad \text { ord } \check{q}_{n, m}^{(2)}=5 .
$$

Функция $\check{q}_{n, m}^{(0)}$ зависит от трех переменных, поэтому мы можем уменьшить их число и получить

$$
\breve{p}_{n, m}^{(0)}=\ln \left(\frac{u_{n, m}-u_{n, m+1}}{u_{n, m}+u_{n, m+1}}\right)^{2}, \quad \breve{q}_{n, m}^{(0)}=2 \ln h_{n, m} .
$$

Дальнейшего упрощения можно достичь, вводя зависящий от $n$ квадратный корень из единицы:

$$
\bar{p}_{n, m}^{(0)}=\ln \left((-1)^{n} \frac{u_{n, m}-u_{n, m+1}}{u_{n, m}+u_{n, m+1}}\right), \quad \bar{q}_{n, m}^{(0)}=\ln h_{n, m} .
$$

Отметим, что можно перейти от закона сохранения (34) к (37), применяя оператор $\left(T_{n}+1\right)^{-1}$ к обеим его частям и принимая во внимание, что $(-1)^{n}$ принадлежит ядру оператора $T_{n}+1$.

Таким образом, мы можем построить законы сохранения в направлении $m$ любого натурального порядка, а в случае направления $n$ мы получаем законы сохранения нечетного порядка. Законы сохранения четного порядка, по-видимому, не существуют, и мы можем доказать это в случае порядка 2. Более точно, мы рассматриваем сохраняющуюся плотность вида $q_{n, m}\left(u_{n, m}, u_{n+1, m}, u_{n+2, m}\right)$ и допускаем явную зависимость от $n, m$. Соответствующая функция $p_{n, m}$ должна зависеть от $u_{n, m}, u_{n+1, m}, u_{n, m+1}$. Мы используем наиболее слабое предположение, что $\partial^{2} q_{n, m} / \partial u_{n, m} \partial u_{n+2, m} \neq 0$ хотя бы в одной точке $n, m$, и доказываем, что такого закона сохранения не существует. 


\section{4. МАСТЕР-СИММЕТРИЯ И ОПЕРАТОР РЕКУРСИИ ДЛЯ ВЫСШИХ СИММЕТРИЙ}

В этом разделе мы обсуждаем проблему построения высших симметрий для дискретного уравнения (1). Имеются две иерархии симметрий в направлениях $n$ и $m$. Рассмотрим сначала случай направления $m$ и построим симметрии, аналогичные (2).

Как отмечалось выше, симметрия (2) эквивалентна известному уравнению (15) типа уравнения Вольтерра. Уравнения, аналогичные (15), обладают мастер-симметриями, которые генерируют для них высшие симметрии и законы сохранения [12]. Более подробное обсуждение можно найти в работах [2], [15], работа [2] содержит конкретные примеры. Здесь мы всего лишь переписываем известную мастер-симметрию уравнения (15) в переменных уравнения (2).

Для мастер-симметрии такого рода сначала мы должны ввести обобщение уравнения (2), зависящее от параметра $\tau$, который будет играть роль времени мастерсимметрии:

$$
\begin{aligned}
\frac{d}{d t_{2}^{(1)}} u_{n, m}= & \frac{(-1)^{n}}{\operatorname{ch} \tau} \frac{u_{n, m}^{2}+u_{n, m-1} u_{n, m+1}}{u_{n, m-1}+u_{n, m+1}}+ \\
& +\operatorname{th} \tau \frac{u_{n, m}\left(u_{n, m+1}-u_{n, m-1}\right)}{u_{n, m-1}+u_{n, m+1}}=\Psi_{n, m}^{(1)}(\tau) .
\end{aligned}
$$

Уравнение (38) при $\tau=0$ совпадает с уравнением (2). Соответствующая мастерсимметрия имеет вид

$$
\frac{d}{d \tau} u_{n, m}=m \Psi_{n, m}^{(1)}(\tau)=\Psi_{n, m}^{*}
$$

Она порождает иерархию симметрий

$$
\frac{d}{d t_{2}^{(j)}} u_{n, m}=\Psi_{n, m}^{(j)}(\tau)
$$

в соответствии с формулой

$$
\begin{aligned}
\Psi_{n, m}^{(j+1)}(\tau) & =\left[\Psi_{n, m}^{*}, \Psi_{n, m}^{(j)}(\tau)\right]=D_{\tau} \Psi_{n, m}^{(j)}(\tau)-D_{t_{2}^{(j)}} \Psi_{n, m}^{*}= \\
& =\frac{\partial \Psi_{n, m}^{(j)}(\tau)}{\partial \tau}+\sum_{k \in \mathbb{Z}}\left(\Psi_{n, m+k}^{*} \frac{\partial \Psi_{n, m}^{(j)}(\tau)}{\partial u_{n, m+k}}-\Psi_{n, m+k}^{(j)}(\tau) \frac{\partial \Psi_{n, m}^{*}}{\partial u_{n, m+k}}\right) .
\end{aligned}
$$

Для всех уравнений (40) величина $\tau$ является внешним параметром, и эти уравнения совместны для любого значения этого параметра. По этой причине мы можем положить $\tau=0$ в уравнениях (40) и получаем иерархию высших симметрий для уравнения (2). Полученные уравнения являются высшими симметриями также и для дискретного уравнения (1).

Например, при $j=2, \tau=0$

$$
\frac{d}{d t_{2}^{(2)}} u_{n, m}=\Psi_{n, m}^{(2)}(0)=\frac{\left(u_{n, m+2}-u_{n, m-2}\right)\left(u_{n, m+1}^{2}-u_{n, m}^{2}\right)\left(u_{n, m}^{2}-u_{n, m-1}^{2}\right)}{\left(u_{n, m}+u_{n, m-2}\right)\left(u_{n, m+1}+u_{n, m-1}\right)^{2}\left(u_{n, m+2}+u_{n, m}\right)} .
$$

Прямым вычислением можно проверить, что это уравнение совместно не только с (2), но и с дискретным уравнением (1). 
В случае направления $n$ мы используем оператор рекурсии для построения высших симметрий. Уравнение (3) эквивалентно известной системе (8), для которой оператор рекурсии был построен в работе [26]. Используя соотношения (9), мы только переписываем этот оператор в скалярном виде, пригодном для уравнения (3).

В этом случае удобно строить оператор рекурсии $R$ в виде

$$
R=H \circ S,
$$

где оператор $H$ является гамильтоновым, а $S$ - симплектический оператор. Эти операторы имеют вид

$$
\begin{aligned}
S= & (-1)^{n}\left(\frac{1}{h_{n, m}} T_{n}+\frac{1}{h_{n-1, m}} T_{n}^{-1}\right) \\
H= & h_{n, m} h_{n-1, m}\left(c_{n} u_{n+2, m}-c_{n-1} u_{n-2, m}\right)\left(T_{n}-1\right)^{-1}(-1)^{n} u_{n, m}+ \\
& +(-1)^{n} u_{n, m} T_{n}\left(T_{n}-1\right)^{-1} h_{n, m} h_{n-1, m}\left(c_{n} u_{n+2, m}-c_{n-1} u_{n-2, m}\right)- \\
& -(-1)^{n} h_{n-1, m} h_{n, m}\left(c_{n} h_{n+1, m} T_{n}+c_{n-1} h_{n-2, m} T_{n}^{-1}\right),
\end{aligned}
$$

где $c_{n}$ - произвольная двухпериодическая функция, зависящая от $n$.

Эти операторы удовлетворяют уравнениям

$$
\begin{gathered}
\frac{d S}{d t_{1}}+S \circ f_{n, m}^{*}+f_{n, m}^{* \perp} \circ S=0, \\
\frac{d H}{d t_{1}}=f_{n, m}^{*} \circ H+H \circ f_{n, m}^{* \perp} .
\end{gathered}
$$

Здесь $f_{n, m}^{*}, f_{n, m}^{* \perp}$ - операторы, определяемые правой частью $f_{n, m}$ уравнения (3):

$$
\frac{d}{d t_{1}} u_{n, m}=f_{n, m} .
$$

Дискретный аналог производной Фреше $f_{n, m}^{*}$ функции $f_{n, m}$ имеет вид

$$
f_{n, m}^{*}=\sum_{k=-2}^{2} \frac{\partial f_{n, m}}{\partial u_{n+k, m}} T_{n}^{k},
$$

а сопряженный ему оператор $f_{n, m}^{* \perp}$ определяется формулой

$$
f_{n, m}^{* \perp}=\sum_{k=-2}^{2} \frac{\partial f_{n+k, m}}{\partial u_{n, m}} T_{n}^{k} .
$$

Из (44) следует, что оператор $R=H \circ S$ удовлетворяет следующему уравнению Лакса:

$$
\frac{d R}{d t_{1}}=\left[f_{n, m}^{*}, R\right]
$$

где $[A, B]=A \circ B-B \circ A$. Все эти формулы стандартны и могут быть найдены, например, в работе [15] для случая автономных уравнений (45) и в работе [13] для неавтономного случая. 
Уравнения (44) и (46) можно считать определениями симплектического, гамильтонова и рекурсионного операторов (см., например, [25]). Гамильтонов оператор $H$ и симплектический оператор $S$ обеспечивают связь между законами сохранения и высшими симметриями уравнения (3). Оператор $R$, удовлетворяющий (46), позволяет строить законы сохранения и высшие симметрии уравнения (3). Например, из (46) мы получаем, что

$$
\frac{\partial}{\partial t_{1}^{(k)}} u_{n, m}=R^{k-1}\left(f_{n, m}\right)=f_{n, m}^{(k)}, \quad k \geqslant 2,
$$

являются высшими симметриями уравнений (3), (45). В случае $k=2$

$$
\begin{aligned}
f_{n, m}^{(2)}= & \hat{f}_{n, m}^{(2)}-\left(c_{n}+c_{n-1}\right) f_{n, m}+\left(c_{n} a_{n-1}-c_{n-1} a_{n}\right)(-1)^{n} u_{n, m}, \\
\hat{f}_{n, m}^{(2)}= & h_{n, m} h_{n-1, m}\left(b_{n} h_{n+1, m} h_{n+2, m} u_{n+4, m}-b_{n-1} h_{n-2, m} h_{n-3, m} u_{n-4, m}+\right. \\
& +u_{n, m}\left(b_{n} u_{n+2, m} h_{n-2, m} u_{n-3, m}-b_{n-1} u_{n-2, m} h_{n+1, m} u_{n+3, m}\right)+ \\
& +\left(u_{n-1, m} h_{n, m}-u_{n+1, m}\right)\left(b_{n} u_{n+2, m}^{2}-b_{n-1} u_{n-2, m}^{2}\right)- \\
& \left.-u_{n, m}\left(b_{n} u_{n-1, m} u_{n+2, m}-b_{n-1} u_{n+1, m} u_{n-2, m}\right)\right),
\end{aligned}
$$

где $b_{n}=a_{n} c_{n}$. Эта симметрия была найдена в работе [1]. Можно проверить, что она также является высшей симметрией дискретного уравнения (1).

По формуле (47) строятся симметрии вида

$$
\frac{\partial}{\partial t_{1}^{(k)}} u_{n, m}=f_{n, m}^{(k)}\left(u_{n+2 k, m}, u_{n+2 k-1, m}, \ldots, u_{n-2 k+1, m}, u_{n-2 k, m}\right),
$$

которые можно назвать симметриями четных порядков $2 k$. В работе [1] было показано, что симметрии первого порядка не существует. Вероятно, в этом случае не существует высших симметрий нечетных порядков.

Мы видим, что высшие симметрии для уравнений (3) и (1) зависят от произвольных двухпериодических функций переменной $n$. То же самое верно для гамильтонова и рекурсионного операторов. Такая ситуация необычна в случае скалярных дискретно-дифференциальных уравнений типа (3) и, вероятно, возникает впервые. Поэтому систему Цучиды (8) можно считать аналогом релятивистской цепочки Тоды, у которой симметрии и эти операторы также зависят от двух параметров. Подобные свойства симметрий обсуждались в работе [12], а свойства операторов исследовались в [27].

\section{5. ГИПЕРБОЛИЧЕСКИЕ СИСТЕМЫ УРАВНЕНИЙ}

В этом разделе мы получаем две интегрируемые гиперболические системы уравнений вместе с $L-A$-парами из высших симметрий дискретного уравнения (1).

Рассмотрим две совместные симметрии вида (3) с $a_{n}=\chi_{n}$ и $a_{n}=\chi_{n-1}$, где $\chi_{n}=\left(1+(-1)^{n}\right) / 2$, а именно уравнения

$$
\begin{aligned}
& \partial_{x} u_{n, m}=h_{n, m} h_{n-1, m}\left(\chi_{n} u_{n+2, m}-\chi_{n-1} u_{n-2, m}\right), \\
& \partial_{y} u_{n, m}=h_{n, m} h_{n-1, m}\left(\chi_{n-1} u_{n+2, m}-\chi_{n} u_{n-2, m}\right) .
\end{aligned}
$$


Из (11), (13) можно получить следующие системы линейных уравнений:

$$
D_{x} \Psi_{n, m}=A_{n, m}^{(1)} \Psi_{n, m}, \quad D_{y} \Psi_{n, m}=A_{n, m}^{(2)} \Psi_{n, m},
$$

где $A_{n, m}^{(1)}$ и $A_{n, m}^{(2)}$ совпадают с матрицей $A_{n, m}$, в которую подставлено $a_{n}=\chi_{n}$ и $a_{n}=\chi_{n-1}$ соответственно. Эта система линейных уравнений совместна на решениях системы (49). Теперь мы изменяем матрицы $A_{n, m}^{(1)}$ и $A_{n, m}^{(2)}$, выражая функции $u_{n \pm 2, m}$ через $u_{n, m}, u_{n \pm 1, m}$, а также $\partial_{x} u_{n, m}$ или $\partial_{y} u_{n, m}$. Мы делаем это, используя следствия системы (49)

$$
\begin{aligned}
\chi_{n} u_{n+2, m} & =\frac{\chi_{n} \partial_{x} u_{n, m}}{h_{n, m} h_{n-1, m}}, & \chi_{n-1} u_{n-2, m} & =-\frac{\chi_{n-1} \partial_{x} u_{n, m}}{h_{n, m} h_{n-1, m}}, \\
\chi_{n-1} u_{n+2, m} & =\frac{\chi_{n-1} \partial_{y} u_{n, m}}{h_{n, m} h_{n-1, m}}, & \chi_{n} u_{n-2, m} & =-\frac{\chi_{n} \partial_{y} u_{n, m}}{h_{n, m} h_{n-1, m}} .
\end{aligned}
$$

В результате мы получаем матрицы, которые зависят только от $u_{n, m}, u_{n+1, m}, u_{n-1, m}$, и, используя эти матрицы, мы можем получить из (50) систему трех уравнений для трех неизвестных функций.

Чтобы избежать явной зависимости от $n$, следует перейти к четным или нечетным $n$. В случае нечетных $n=2 k-1$ введем следующие обозначения:

$$
p=u_{2 k-1, m}, \quad q=u_{2 k, m}, \quad r=u_{2 k-2, m} .
$$

Тогда матрицы примут вид

$$
\begin{aligned}
A^{(1)} & =\left(\begin{array}{cc}
\frac{q p_{x}}{1-p q}+\frac{2 \lambda}{\lambda-1} & -q \\
\frac{2 \lambda p_{x}}{(\lambda-1)(1-p q)} & 1-p q
\end{array}\right), \\
A^{(2)} & =\left(\begin{array}{cc}
\frac{(\lambda-1)(p r-1)}{1+\lambda} & \frac{(1-\lambda) r}{1+\lambda} \\
\frac{2 \lambda p(p r-1)}{1+\lambda} & \frac{r p_{y}}{p r-1}-\frac{2 \lambda p r}{1+\lambda}
\end{array}\right) .
\end{aligned}
$$

Соответствующие линейные уравнения можно записать в виде

$$
D_{x} \Psi=A^{(1)} \Psi, \quad D_{y} \Psi=A^{(2)} \Psi,
$$

и их условие совместности выглядит следующим образом:

$$
D_{x} A^{(2)}-D_{y} A^{(1)}=\left[A^{(1)}, A^{(2)}\right] .
$$

Это матричное уравнение эквивалентно гиперболической системе

$$
\begin{array}{r}
\frac{\partial^{2} \ln p}{\partial x \partial y}+\frac{p_{x} p_{y}}{p^{2}(p q-1)(p r-1)}+(p q-1)(p r-1)=0, \\
(p r-1) q_{y}+q r p_{y}-r(p q-1)(p r-1)=0, \\
(p q-1) r_{x}+q r p_{x}+q(p q-1)(p r-1)=0 .
\end{array}
$$


Таким образом, мы получили интегрируемую систему (56) трех гиперболических уравнений вместе с $L-A$-парой $(53)-(55)$. Если $u_{n, m}(x, y)$ - общее решение уравнений (49), то функции (52) удовлетворяют системе (56) при любых $k, m$.

При четных значениях $n=2 k$ мы получаем ту же самую гиперболическую систему с точностью до инволюции $x \leftrightarrow y$. В обоих случаях, независимо от четности $n$, первое из уравнений (56) может быть записано в виде

$$
\begin{aligned}
\frac{\partial^{2} \ln u_{n, m}}{\partial x \partial y} & +\frac{\partial_{x} u_{n, m} \partial_{y} u_{n, m}}{u_{n, m}^{2}\left(u_{n, m} u_{n-1, m}-1\right)\left(u_{n, m} u_{n+1, m}-1\right)}+ \\
& +\left(u_{n, m} u_{n-1, m}-1\right)\left(u_{n, m} u_{n+1, m}-1\right)=0,
\end{aligned}
$$

и это - не что иное, как $(2+1)$-мерная цепочка $(m-$ внешний параметр), подобная $(2+1)$-мерному обобщению цепочки Тоды [17]. Любое совместное решение $u_{n, m}(x, y)$ уравнений (49) является также решением цепочки (57). Вопрос об интегрируемости цепочки (57) в настоящей работе не исследовался и остается открытым.

Для получения второй гиперболической системы уравнений рассматривается следующая пара высших симметрий:

$$
\begin{aligned}
& \partial_{x} u_{n, m}=h_{n, m} h_{n-1, m}\left(\chi_{n} u_{n+2, m}-\chi_{n-1} u_{n-2, m}\right), \\
& \partial_{z} u_{n, m}=(-1)^{n} \frac{u_{n, m}^{2}+u_{n, m+1} u_{n, m-1}}{u_{n, m+1}+u_{n, m-1}},
\end{aligned}
$$

совместная на решениях дискретного уравнения (1). Соответствующая вспомогательная линейная задача имеет вид

$$
D_{x} \Psi_{n, m}=A_{n, m}^{(1)} \Psi_{n, m}, \quad D_{z} \Psi_{n, m}=B_{n, m} \Psi_{n, m} .
$$

Здесь матрица $A_{n, m}^{(1)}$ определена, как в предыдущем случае, матрица $B_{n, m}$ задается формулой (20).

Матрица $A_{n, m}^{(1)}$ преобразовывается при помощи формул $(51)$, в матрице $B_{n, m}$ мы исключаем $u_{n, m-1}$, используя второе из уравнений (58). Для устранения явной зависимости от $n$ перейдем к нечетным $n=2 k-1$ и введем обозначения

$$
p=u_{2 k-1, m}, \quad q=u_{2 k, m}, \quad r=u_{2 k-1, m+1} .
$$

В результате мы получаем ту же самую матрицу $A^{(1)}(53)$ и следующую матрицу $B$ :

$$
B=\frac{1}{p-r}\left(\begin{array}{cc}
(\lambda-1)\left(p_{z}+p\right) & \frac{(1-\lambda)\left(p_{z}+r\right)}{p+r} \\
\lambda(p+r)\left(p_{z}+p\right) & \frac{(p-r)\left(p_{z}-p\right)}{p+r}-\lambda\left(p_{z}+r\right)
\end{array}\right) .
$$

В этом случае вместо (55) мы приходим к матричному соотношению

$$
D_{x} B-D_{z} A^{(1)}=\left[A^{(1)}, B\right] .
$$

Соотношение (60) эквивалентно следующей гиперболической системе:

$$
\begin{aligned}
\frac{\partial^{2} \ln p}{\partial x \partial z}+\frac{\left(p_{z}-p\right)(p-r) p_{x}}{p^{2}(p+r)(p q-1)}+\frac{\left(p_{z}+p\right)(p+r)(p q-1)}{(p-r) p} & =0 \\
\left(p^{2}-r^{2}\right) q_{z}-2(q r-1) p_{z}-q\left(p^{2}+r^{2}\right)+2 r & =0 \\
(p q-1) r_{x}-(q r-1) p_{x}-(p+r)(p q-1)(q r-1) & =0 .
\end{aligned}
$$


Имеются четыре варианта гиперболических систем в зависимости от выбора четного или нечетного $n$ и исключения функции $u_{n, m+1}$ или $u_{n, m-1}$ в матрице $B_{n, m}$. Однако все эти системы являются эквивалентными с точностью до простых точечных преобразований.

Случай высших симметрий (49), совместных друг с другом без дополнительных предположений, аналогичен примерам, рассмотренным в работах [12], [28]. Второй случай высших симметрий (58), которые совместны на решениях дискретного уравнения (1), по-видимому, является новым.

Во втором случае мы также можем построить $(2+1)$-мерную цепочку, подобную (57). Однако она является неавтономной и весьма громоздкой, и по этим причинам мы ее не выписываем.

В системах (56), (61) мы можем исключить $r$ или $q$, используя соответственно второе или третье уравнение. В этом случае мы получим гиперболические системы двух уравнений, аналогичные системам, представленным в работах [12], [28]. Однако эти системы содержат квадратные корни и имеют сложный вид.

\section{6. ЗАКЛЮЧЕНИЕ}

В настоящей работе мы построили $L-A$-пару для дискретного уравнения (1) и продемонстрировали, что это уравнение отличается от известных примеров с точки зрения $L-A$-пар.

Большинство известных интегрируемых дискретных уравнений вида (6) имеет $L-A$-пару вида

$$
\left(T_{n}-N_{n, m}\right) \Psi_{n, m}=0, \quad\left(T_{m}-M_{n, m}\right) \Psi_{n, m}=0
$$

с $(2 \times 2)$-матрицами $N_{n, m}, M_{n, m}$. Имеются также дискретные аналоги уравнения Цицейки с $(3 \times 3)$-матрицами в $L-A$-паре $(62) . L-A$-пара нашего уравнения (1) имеет вид

$$
\left(T_{n}^{2}-N_{n, m}\right) \Psi_{n, m}=0, \quad\left(T_{m}-M_{n, m}\right) \Psi_{n, m}=0
$$

c $(2 \times 2)$-матрицами.

В разделе 4 мы построили для уравнения (1) две иерархии высших симметрий в обоих направлениях $m$ и $n$. Эти иерархии оказываются разнотипными: в направлении $m$ имеются симметрии любого натурального порядка, тогда как в направлении $n$ мы имеем только симметрии четного порядка. Для других известных примеров обе иерархии однотипны.

Иерархии законов сохранения, построенные в разделе 3, также оказываются разнотипными. В направлении $m$ существуют законы сохранения любого натурального порядка, а в направлении $n$ - только нечетного порядка.

Уравнение (3) с $a_{n}=1$ относится к классу автономных уравнений вида (22) и является младшим членом своей иерархии. Можно было ожидать, что оно окажется аналогом цепочки Ито-Нариты-Богоявленского (24). Наше исследование показало, что уравнение (3) с $a_{n}=1$ тесно связано с известной интегрируемой системой Цучиды (8), которая по своим симметрийным свойствам близка к релятивистской цепочке Тоды. 
Другие известные интегрируемые цепочки вида (22) имеют $L$-A-пары (23) с $(2 \times 2)$ - или $(3 \times 3)$-матрицами. Наш пример уравнения $(3)$ с $a_{n}=1$ отличается от них тем, что $L-A$-пара имеет вид $(25)$ с матрицами размера $2 \times 2$.

Благодарности. Работа поддержана РФФИ (гранты № 12-01-31208, 13-01-00070, 14-01-97008-р-поволжье_а) и Министерством образования и науки РФ (соглашение $8499)$.

\section{Список литературы}

[1] R. N. Garifullin, R. I. Yamilov, J. Phys. A, 45:34 (2012), 345205, 23 pp.

[2] D. Levi, M. Petrera, C. Scimiterna, R. Yamilov, SIGMA, 4 (2008), 077, 14 pp.

[3] D. Levi, R. I. Yamilov, J. Phys. A, 44:14 (2011), 145207, 22 pp., arXiv: 1011.0070.

[4] P. Xenitidis, "Integrability and symmetries of difference equations: the Adler-BobenkoSuris case", Group Analysis of Differential Equations and Integrable Systems (Protaras, Cyprus, October 26-30, 2008), University of Cyprus, Nicosia, 2009, 226-242, arXiv: 0902.3954.

[5] P. D. Xenitidis, V. G. Papageorgiou, J. Phys. A, 42:45 (2009), 454025, 13 pp.

[6] V.E. Adler, "On a discrete analog of the Tzitzeica equation", arXiv: 1103.5139.

[7] A. V. Mikhailov, P. Xenitidis, Lett. Math. Phys., 104:4 (2013), 431-450, arXiv: 1305.4347.

[8] C. Scimiterna, M. Hay, D. Levi, On the integrability of a new lattice equation found by multiple scale analysis, arXiv: 1401.5691.

[9] T. Tsuchida, J. Phys. A, 35:36 (2002), 7827-7847, arXiv: nlin/0105053.

[10] M. J. Ablowitz, A. Ramani, H. Segur, J. Math. Phys., 21:5 (1980), 1006-1015.

[11] V.S. Gerdjikov, M. I. Ivanov, Bulgar. J. Phys., 10:2 (1983), 130-143.

[12] В. Э. Адлер, А. Б. Шабат, Р. И. Ямилов, ТМФ, 125:3 (2000), 355-424.

[13] D. Levi, R. Yamilov, J. Math. Phys., 38:12 (1997), 6648-6674.

[14] Р. И. Ямилов, УМН, 38:1(229) (1983), 155-156.

[15] R. Yamilov, J. Phys. A, 39:45 (2006), R541-R623.

[16] G. Tzitzéica, Rendiconti del Circolo Matematico di Palermo, 25:1 (1907), 180-187.

[17] А. В. Михайлов, Писъма в ЖЖЭТФ, 30:7 (1979), 443-448.

[18] O. I. Bogoyavlensky, Phys. Lett. A, 134:1 (1988), 34-38.

[19] Y. Itoh, Proc. Japan Acad., 51 (1975), 374-379.

[20] K. Narita, J. Phys. Soc. Japan, 51:5 (1982), 1682-1685.

[21] A.V. Mikhailov, "Formal diagonalisation of Darboux transformation and conservation laws of integrable PDEs, PD $\Delta \mathrm{Es}$, and P $\Delta \mathrm{Es",} \mathrm{Доклад} \mathrm{на} \mathrm{Международном}$ научном семинаре "Геометрические структуры в интегрируемых системах" (Москва, МГУ, 30 октября-2 ноября 2012), 2012; http://www.mathnet.ru/php/ presentation.phtml?option lang $=$ eng\&presentid $=5934$.

[22] И. Т. Хабибуллин, М. В. Янгубаева, ТМФ, 177:3 (2013), 441-467.

[23] В. Вазов, Асимптотические разложения решений обыкновенных дифференциальных уравнений, Мир, М., 1968.

[24] В. Г. Дринфельд, В. В. Соколов, “Алгебры Ли и уравнения типа Кортевега-де Фриза”, Итоги науки и техн. Сер. Соврем. пробл. матем. Нов. достиж., 24, ВИНИТИ, М., 1984, $81-180$.

[25] А. В. Михайлов, Дж. П. Ванг, П. Ксенитидис, ТМФ, 167:1 (2011), 23-49.

[26] Ф. Ханизаде, А. В. Михайлов, Дж. П. Ванг, ТМФ, 177:3 (2013), 387-440.

[27] Р. И. Ямилов, ТМФ, 151:1 (2007), 66-80.

[28] А.Б. Шабат, Р. И. Ямилов, Алгебра и анализ, 2:2 (1990), 183-208. 